\title{
Refractive Index Sensor Based on Fano Resonances in Plasmonic Waveguide With Dual Side-Coupled Ring Resonators
}

\author{
Xuewei ZHANG, Yunping QI*, Peiyang ZHOU, Hanhan GONG, \\ Bingbing HU, and Chunman YAN \\ Engineering Research Center of Gansu Province for Intelligent Information Technology and Application, College of \\ Physics and Electronic Engineering, Northwest Normal University, Lanzhou 730070, China \\ "Corresponding author: Yunping QI E-mail: yunpqi@126.com
}

\begin{abstract}
A refractive index sensor based on Fano resonances in metal-insulator-metal (MIM) waveguides coupled with rectangular and dual side rings resonators is proposed. The sensing properties are numerically simulated by the finite element method (FEM). For the interaction of the narrow-band spectral response and the broadband spectral response caused by the side-coupled resonators and the rectangular resonator, respectively, the transmission spectra exhibit a sharp and asymmetric profile. Results are analyzed using the coupled-mode theory based on the transmission line theory. The coupled mode theory is employed to explain the Fano resonance effect. The results show that with an increase in the refractive index of the fill dielectric material in the slot of the system, the Fano resonance peak exhibits a remarkable red shift. Through the optimization of structural parameters, we achieve a theoretical value of the refractive index sensitivity (S) as high as $1160 \mathrm{~nm} / \mathrm{RIU}$, and the corresponding sensing resolution is $8.62 \times 10^{-5}$ RIU. In addition, the coupled MIM waveguide structure can be easily extended to other similar compact structures to realize the sensing task and integrated with other photonic devices at the chip scale. This work paves the way toward the sensitive nanometer scale refractive index sensor for design and application.
\end{abstract}

Keywords: Refractive index sensor; Fano resonances; surface plasmon polaritons; MIM waveguides

Citation: Xuewei ZHANG, Yunping QI, Peiyang ZHOU, Hanhan GONG, Bingbing HU, and Chunman YAN, "Refractive Index Sensor Based on Fano Resonances in Plasmonic Waveguide With Dual Side-Coupled Ring Resonators," Photonic Sensors, 2018, 8(4): $367-374$.

\section{Introduction}

Continuous improvements in nanofabrication and nanocharacterization capabilities have changed the projections about the role that metals could play in the development of new optical devices [1]. As a result, the surface plasmon polaritons (SPPs) are electromagnetic waves that propagate in metals and dielectric interfaces [2-5]. They can be laterally confined below the diffraction limit using subwavelength metal structures, rendering them attractive for the development of miniaturized optical devices [6]. Therefore, SPPs constitute a new subject that has attracted considerable attention in the related fields [7], such as biosensing [8], SPP lithography [9], and optical and ultrahigh resolution

Received: 21 May 2018 / Revised: 9 July 2018

(C) The Author(s) 2018. This article is published with open access at Springerlink.com

DOI: $10.1007 / \mathrm{s} 13320-018-0509-6$

Article type: Regular 
imaging [10].

Among the SPP waveguides, metal-insulatormetal (MIM) waveguides coupled with resonators have flourished and captured the interest of researchers because they can be easily integrated at the chip scale [11]. Recently, MIM plasmonic waveguides offering very high optical confinement and closer spacing to adjacent waveguides or structures have been proposed for diverse applications, such as MIM optical filers [12, 13], electromagnetically induced transparency $[14,15]$, Bragg gratings [16], and directional couplers [17]. Compared with the other sensors, plasmonic sensors with MIM structures have an inherent advantage to achieve high integration. Therefore, many photonic devices based on Fano resonances have been designed by using the coupling effect between narrow dark modes and broad bright modes and have been used in plasmonic sensors [18]. Huang et al. reported a high angular sensitivity of over $500 \mathrm{deg} / \mathrm{RIU}$ [19]. Another design of the plasmonic sensor is based on plasmonic gold mushroom arrays, and the refractive index sensitivity is $1050 \mathrm{~nm} / \mathrm{RIU}$ [20]. Tang et al. reported a refractive index sensor of metal-insulator-metal waveguides coupled with resonators, and the refractive index sensitivity is $1125 \mathrm{~nm} / \mathrm{RIU}$ [21]. Wei et al. reported a plasmonic circular resonator for refractive index sensors and filters, and the refractive index sensitivity is $1010 \mathrm{~nm} / \mathrm{RIU}$ [22]. However, plasmonic sensors currently present low sensitivity, which remains a huge challenge for researchers.

In this paper, a structure of two MIM waveguides coupled with rectangular and dual side-coupled circular ring resonators is proposed for plasmonic refractive index sensors. Compared with other plasmonic sensors, the advantage of this structure is of high sensitivity. The sensitivity of this structure is $1160 \mathrm{~nm} / \mathrm{RIU}$, larger than the reported plasmonic device to date [21, 22].

\section{Model structure and analytical method}

A schematic of the proposed refractive index sensor is shown in Fig. 1. The sensor is composed of two MIM waveguides, a rectangular resonator, and dual side-coupled circular ring resonators. The gray and white areas represent the silver $\left(\varepsilon_{m}\right)$ layer and dielectric $\left(\varepsilon_{n}\right)$, respectively. The widths of the MIM waveguides and rings cavity are fixed at $w=50 \mathrm{~nm}$ to ensure that only the fundamental transverse magnetic $\left(\mathrm{TM}_{0}\right)$ mode is supported in the MIM waveguides [23]. The input and output ports are in the right and left MIM waveguides. The inner (outer) radii of the two rings are $r_{1}\left(r_{2}\right)$ and $r_{1}{ }^{\prime}\left(r_{2}{ }^{\prime}\right)$, respectively. In Fig. $1, t_{1}$ and $t_{2}$ are the coupling distances between the MIM waveguide and rectangular cavity, and between the rectangular cavity and rings cavity, respectively. Meanwhile, we define $r=\left(r_{1}+r_{2}\right) / 2$ and $r^{\prime}=\left(r_{1}{ }^{\prime}+r_{2}{ }^{\prime}\right) / 2$ as the radii of two ring resonators, and $h$ and $d$ are the height and width of the rectangular cavity, respectively. $n$ is the refractive index of the fill dielectric. The permittivity of Ag can be of Debye-Drude dispersion mode $[23,24]$.

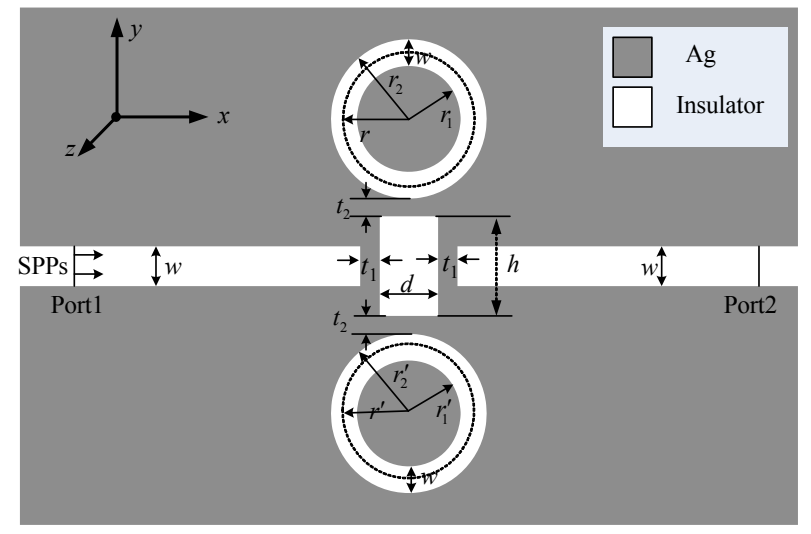

Fig. 1 2-dimensional (2D) schematic of the metal-insulatormetal waveguides coupled with rectangular and two shunt-wound rings resonator.

$$
\varepsilon(\omega)=\varepsilon_{\infty}+\frac{\left(\varepsilon_{s}-\varepsilon_{\infty}\right)}{(1+\mathrm{j} \omega \tau)}+\sigma / \mathrm{j} \omega \varepsilon_{0}
$$

where $\varepsilon_{\infty}=3.8344$ and $\varepsilon_{s}=-9530.5$ are the infinite frequency permittivity and the static permittivity, respectively, $\tau=7.35 \times 10^{-15} \mathrm{~s}$ is the relaxation time, 
and $\sigma=1.1486 \times 10^{7} \mathrm{~S} / \mathrm{m}$ is the conductivity of $\mathrm{Ag}$.

The $\mathrm{TM}_{0}$ model of the MIM waveguide can be expressed as [25]

$$
\tanh (k w)=\frac{-2 k p \alpha_{c}}{\left(k^{2}+p^{2}+\alpha_{c}^{2}\right)}
$$

where $k=2 \pi / \lambda$ is the wave vector in the waveguide, $w$ is the width of each MIM waveguide, $p=\varepsilon_{n} / \varepsilon_{m}$, $\alpha_{c}=\left[k_{0}^{2}\left(\varepsilon_{n}-\varepsilon_{m}\right)+k^{2}\right]^{1 / 2}$, and $k_{0}$ is the wave vector in the free space. The transmission wavelengths can be derived on the basis of the standing wave theory as $[26,27]$

$$
\begin{aligned}
& \lambda_{m}=\frac{2 \operatorname{Re}\left(n_{\text {eff }}\right) L}{m-\Psi_{r} / \pi} \quad(m=1,2, \cdots) \\
& \operatorname{Re}\left(n_{\text {eff }}\right)=\sqrt{\left[\varepsilon_{m}+\left(\frac{k}{k_{0}}\right)^{2}\right]}
\end{aligned}
$$

where $\operatorname{Re}\left(n_{\text {eff }}\right)$ is the real part of the effective refractive index of a wavelength in the MIM waveguide, which can be derived by (4). In (3) and (4), $L$ is the perimeter of the ring cavity, and $\Psi_{r}$ is the phase shift of the beam reflected at one end of the cavity.

In this section, the MIM waveguides coupled with the rectangular and ring cavities are analyzed on the basis of the temporal coupled-mode theory [28]. To explain the Fano resonance phenomenon, we introduce certain parameters, namely, the SPP wave of the cavity $\left(E_{1}, E_{2}\right)$ and the coupling coefficients between the input MIM waveguide and rectangular cavity $\left(k_{1}\right)$, between the output MIM waveguide and the rectangular cavity $\left(k_{2}\right)$, and between the rectangular cavity and rings cavity $\left(k_{r}\right)$. When a certain optical wave with $\omega$ frequency is input on the input port of the waveguide, transmittance $T$ can be expressed as [21,29]

$$
\begin{gathered}
T=\left|\frac{E_{2}}{E_{1}}\right|^{2}= \\
\left|\frac{k_{1} k_{2}\left[\mathrm{j}\left(\omega-\omega_{r}\right)+k_{r}^{2}\right]}{\left[\mathrm{j}\left(\omega-\omega_{s}\right)+k_{1}^{2}+k_{2}^{2}+k_{r}^{2}\right]\left[\mathrm{j}\left(\omega-\omega_{r}\right)+k_{r}^{2}\right]-k_{r}^{2}}\right|^{2}
\end{gathered}
$$

where $\omega$ is the frequency of the incident wave and $\omega=c / \lambda, \omega_{s}$ and $\omega_{r}$ are the resonance frequencies of the rectangular and rings cavities, respectively. In addition, we define FWHM as the full width at half maximum, FWHM $=\lambda_{2}-\lambda_{1}$, where $\lambda_{1}$ and $\lambda_{2}$ are the values on the transmission spectra at which the transmission value is $\left(T_{\max }+T_{\min }\right) / 2 . T_{\max }$ and $T_{\min }$ are the peak and valley values of the transmission, respectively.

The finite element method (FEM) with scattering boundary conditions is employed to simulate and research its sensing characteristics. In the following FEM simulations, the fundamental TM mode of the SPPs is excited by a port on the left slit. The transmission is defined to be $T=P_{\text {out }} / P_{\text {in }}, P_{\text {in }}$ presents the total incident power of Port1, and $P_{\text {out }}$ is the transmission power of Port2. The transmission spectra of the plasmonic sensor are obtained by parametrically sweeping the input wavelength of $\lambda$ with the step of $1 \mathrm{~nm}$. The magnetic field distributions in this structure are analyzed. The relationships between the resonance peaks in the transmission spectra and the refractive index of the material under sensing are analyzed. Additionally, the structural parameters of the sensor impact on sensitivity are analyzed to optimize the performance.

\section{Simulation results and analysis}

In this section, the transmission spectra of the MIM waveguides are simulated under varying parameters of the structure. We fix $r=r^{\prime}=115 \mathrm{~nm}$, $n=1, h=100 \mathrm{~nm}, d=w=50 \mathrm{~nm}$, and $t=10 \mathrm{~nm}$. The light incident from the left end of the straight guide will excite the surface plasmon polaritons in the MIM waveguide and be coupled with both the rectangle and rings resonators. The rectangular resonator excites a wide spectrum resonance mode, and the circular rings cavity resonates a narrow spectrum resonance mode. The coupling between the different resonant modes of the two resonant cavities forms the Fano resonance. Figure 2(a) shows the transmission spectra of the structures with 
one ring cavity, and two shunt-wound rings resonators, and without ring cavity. The structure without a ring cavity exhibits a low transmittance along a linear curve with a negative slope. When the MIM waveguides are coupled with both two rings resonators and a rectangular resonator, a peak and a dip exist in the asymmetrical transmission spectra. The triangle solid shows a Fano resonance in the MIM waveguide. As can be seen from Fig.2(a), the solid curve is obtained by solving (5), which is in good agreement with the simulation results.
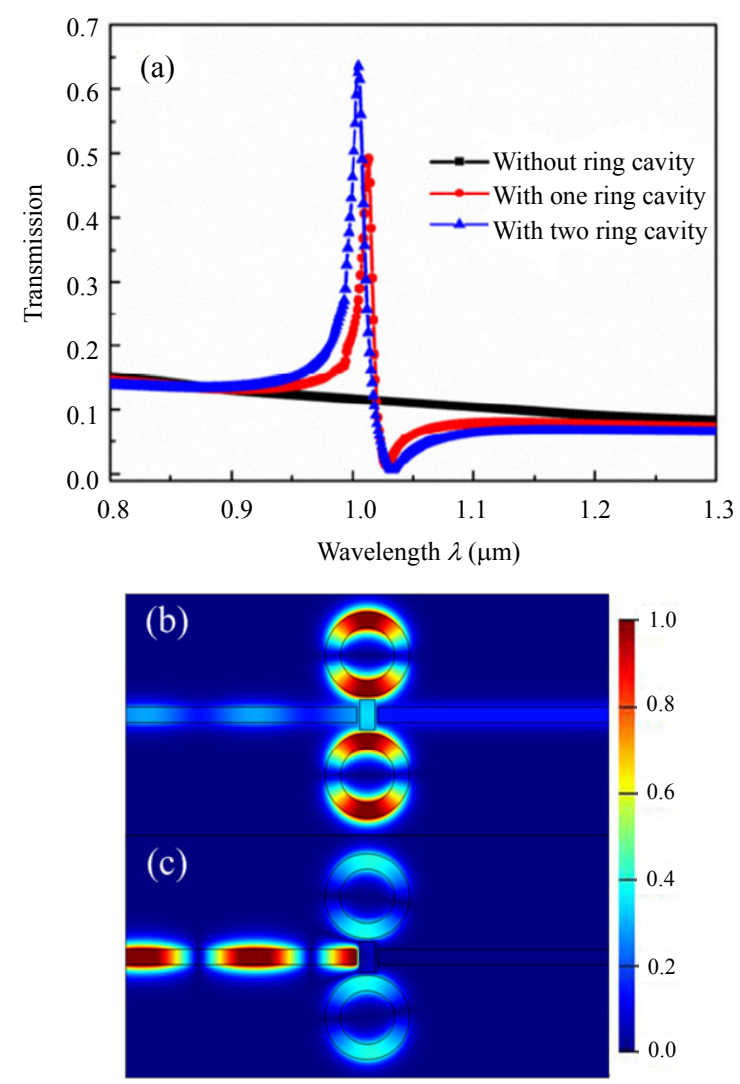

Fig. 2 Transmission spectra of MIM waveguides coupled with (a) one ring, two rings resonant cavities, and by a rectangular cavity only; the contour profiles of field $H_{z}$ of the waveguides coupled with rectangular and two shunt-wound rings resonator at (b) $\lambda=1005 \mathrm{~nm}$ and (c) $\lambda=1033 \mathrm{~nm}$.

Figures 2(b) and 2(c) show the steady-state magnetic field $H_{z}$ distributions at points of the peak and dip of the MIM waveguides coupled with two rings and rectangular cavities. At the peak position $\left(\lambda_{\text {peak }}=1005 \mathrm{~nm}\right)$, an in-phase relationship exists between the lower parts of the rings resonators and rectangular resonator, whereas an anti-phase relationship is evident between the lower and rectangular resonators. However, at the dip position $\left(\lambda_{\text {dip }}=1033 \mathrm{~nm}\right)$, anti-phase relationships exist between the lower parts of the rings resonators and rectangular resonator and between the lower and upper parts of the rings resonators. According to (1) - (4), the effective SPP wavelength, $\lambda_{\mathrm{spp}}=\lambda / \operatorname{Re}\left(n_{\mathrm{eff}}\right)$, for $\lambda=1005 \mathrm{~nm}$ is $718 \mathrm{~nm}$. At $\lambda=1005 \mathrm{~nm}, 2 \pi r / \lambda_{\mathrm{spp}} \approx$ 1 for the rings resonator, and $2(h+d) / \lambda_{\mathrm{spp}} \approx 0.41$ for the rectangular resonator. These results show that $\lambda_{\mathrm{spp}}=718 \mathrm{~nm}$ meets the wave resonance condition of the rings resonator, which is in good agreement with the results of the numerical calculation.

\section{Transmission properties of the proposed sensor with different parameters and discussion}

In a similar way, we provide another way to control the transmission spectra by changing the refractive index $n$ on the MIM waveguide. The refractive index $n$ is set from 1 to 1.1 with a step of 0.02 RIU, and other structural parameters $t, d, h$, and $r$ are fixed at $10 \mathrm{~nm}, 50 \mathrm{~nm}, 100 \mathrm{~nm}$, and $115 \mathrm{~nm}$, respectively. Figures 3(a) and 3(b) show the shifts of the Fano resonance peaks with increasing $n$ and the relationship of the peak shift with $\delta n$, respectively. By changing the refractive index, the transmission spectra exhibit a red shift as shown in Fig.3(a). As can be seen from Fig. 3(b), it is found that the shift of the Fano resonance peaks has a nearly linear relationship with the refractive index $\delta n$. With an increase in $n, \operatorname{Re}\left(n_{\text {eff }}\right)$ increases. The Fano resonance peak shows a red shift as $n$ increases. The sensitivity $(S)$ of the refractive index sensor is $\delta \lambda / \delta n=$ $980 \mathrm{~nm} / \mathrm{RIU}$, the corresponding sensing resolution is $1.02 \times 10^{-4}$ RIU, and the figure of merit (FOM) of the sensor is $F O M=S / F W H M=62$ [30].

The transmission spectra of refractive index 1 for values of $r\left(r^{\prime}\right)$ varying from $95 \mathrm{~nm}$ to $135 \mathrm{~nm}$ are demonstrated in rings resonators, causing the red shift of the Fano resonance peak. Figure 4(a) shows the shift in the Fano resonance peaks as $r$ increases 
simultaneously. The shift in the Fano resonance peaks versus refractive index $\delta n$ of the analyte for different $r$ values is demonstrated in Fig. 4(b). All the Fano resonance peaks increase linearly as the refractive index increases. The sensitivity of the plasmonic refractive index sensor has a positive correlation with incremental $r$ in a range from $\delta \lambda / \delta n=800 \mathrm{~nm} / \mathrm{RIU}(r=95 \mathrm{~nm})$ and its $F O M=50$ to $\delta \lambda / \delta n=1160 \mathrm{~nm} / \mathrm{RIU}(r=135 \mathrm{~nm})$ and its $F O M=73$. The maximum sensitivity is $1160 \mathrm{~nm} / \mathrm{RIU}$, and its corresponding sensing resolution is $8.62 \times 10^{-5}$ RIU. Therefore, the radius of the ring resonator influences not only the Fano resonance peak position but also the sensitivity.

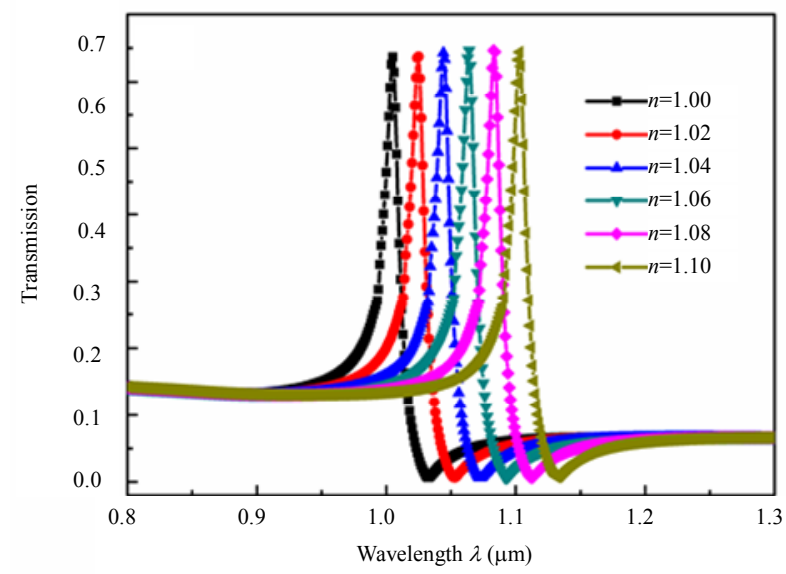

(a)

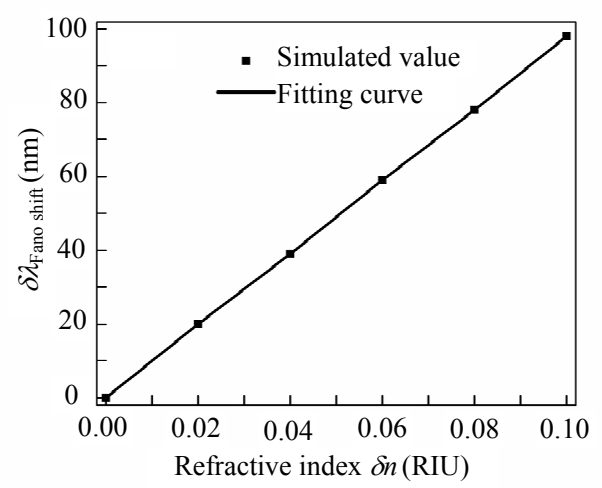

(b)

Fig. 3 Transmission spectra of the MIM waveguide coupled with two rings and rectangular cavities with (a) different refractive indexes $n$ and (b) shift in the Fano resonance peak as a function of refractive index $\delta n$. Here, $r=r^{\prime}=115 \mathrm{~nm}$.

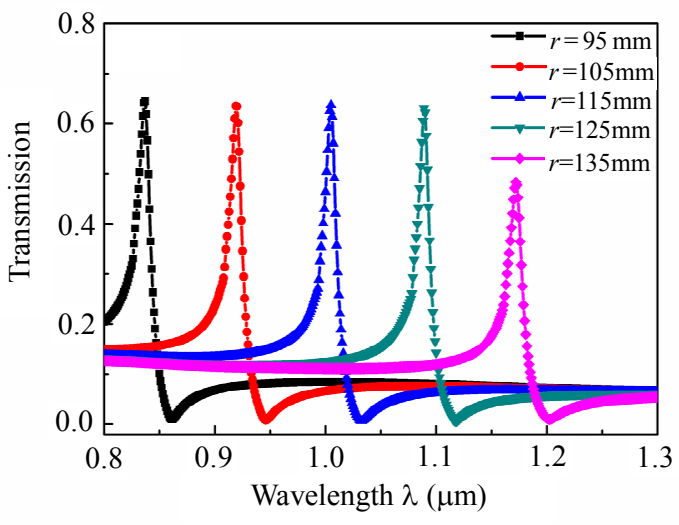

(a)

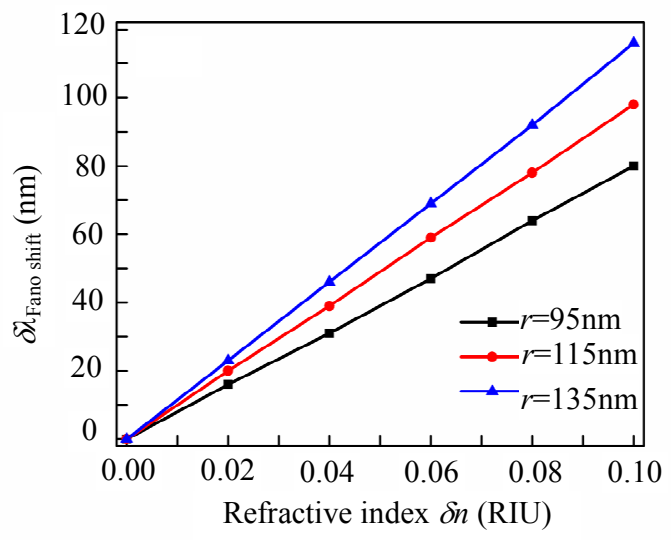

(b)

Fig. 4 Transmission spectra of the MIM waveguide coupled with two rings and rectangular cavities with (a) different $r$ and (b) shift in the Fano resonance peak as a function of the refractive index $\delta n$. Here, $r=r^{\prime}$ and $n=1$.

Next, we analyze the impact of different coupling distances $t$ on sensing properties of the plasmonic refractive index sensor. The transmission spectra of the MIM waveguide coupled with two rings and rectangular cavities for values of $t_{1}\left(t_{2}=\right.$ $10 \mathrm{~nm}$ ) varying from $6 \mathrm{~nm}$ to $14 \mathrm{~nm}$ are demonstrated in Fig. 5(a). Figure 5(a) shows that with an increase in $t_{1}$, the energy confined in the structure increases. The transmission spectra of the MIM waveguide coupled with two rings and rectangular cavities for values of $t_{2}\left(t_{1}=10 \mathrm{~nm}\right)$ varying from $6 \mathrm{~nm}$ to $14 \mathrm{~nm}$ are demonstrated in Fig. 5(b). Figure 5(b) shows that with an increase in $t_{2}$, the energy confined in the structure increases. The resonance wavelengths have a blue shift with increasing of the $t_{2}$. 


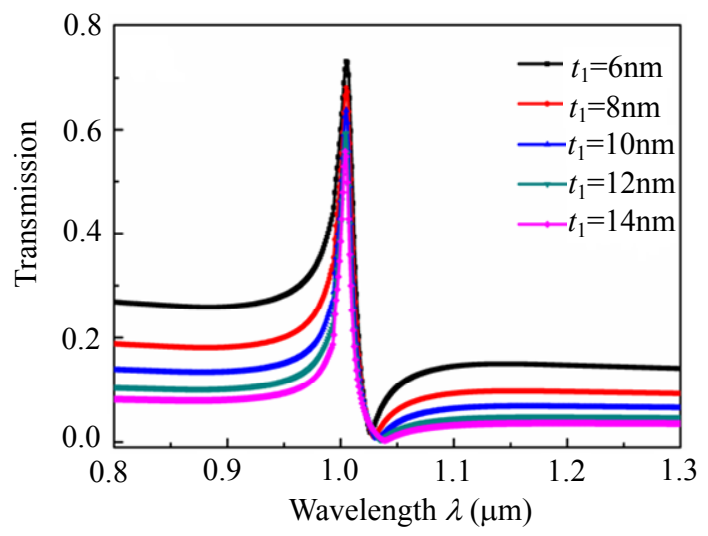

(a)

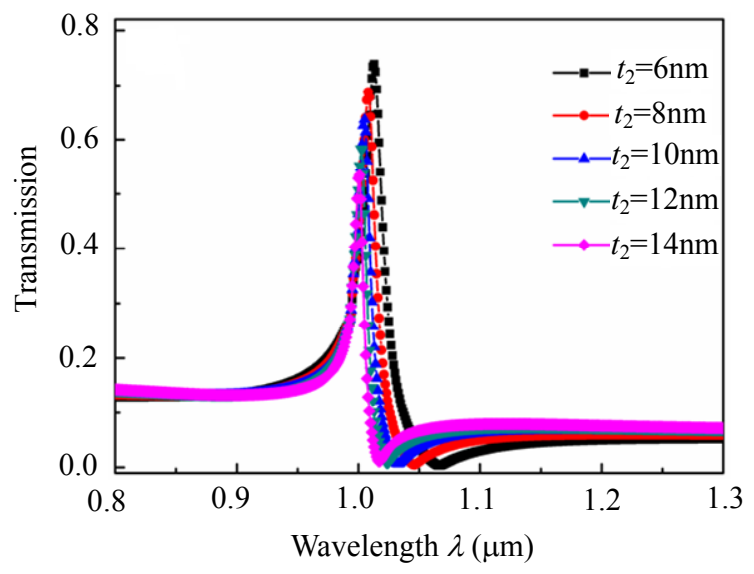

(b)

Fig. 5 Transmission spectra of the MIM waveguide coupled with two rings and rectangular cavities with varying coupling distances (a) $t_{1}\left(t_{2}=10 \mathrm{~nm}\right)$ and (b) $t_{2}\left(t_{1}=10 \mathrm{~nm}\right)$. Here, $r=r^{\prime}=$ $115 \mathrm{~nm}, n=1, d=50 \mathrm{~nm}$, and $h=100 \mathrm{~nm}$.

Furthermore, the effects of the different widths of the rectangular cavity on the Fano resonance of MIM waveguide are studied. The $d$ varies from $30 \mathrm{~nm}$ to $70 \mathrm{~nm}$ at intervals of $20 \mathrm{~nm}$ with $r=r^{\prime}=$ $115 \mathrm{~nm}, n=1, t=10 \mathrm{~nm}$, and $h=100 \mathrm{~nm}$. In Fig. 6(a), it can be seen that with an increase in the width of the rectangular resonator, the transmission spectra have a slightly red shift, and the transmittance gradually decreases. As the width increases, the value of $L$ increases. The effects of the different heights of the rectangular cavity on the Fano resonance of the MIM waveguide are studied. $h$ varies from $60 \mathrm{~nm}$ to $140 \mathrm{~nm}$ at intervals of $20 \mathrm{~nm}$ with $r=r^{\prime}=115 \mathrm{~nm}, n=1, t=10 \mathrm{~nm}$, and $d=50 \mathrm{~nm}$. As can be seen from Fig. 6(b), with an increase in $h$, the transmittance of the light wave decreases gradually, and the position of the Fano resonance peak remains unchanged. With an increase in the height and width of the rectangular cavity, the volume of the rectangular cavity increases, and the energy of the incident light is more restricted in the rectangular and ring resonator, so the transmittance of the exit end decreases.

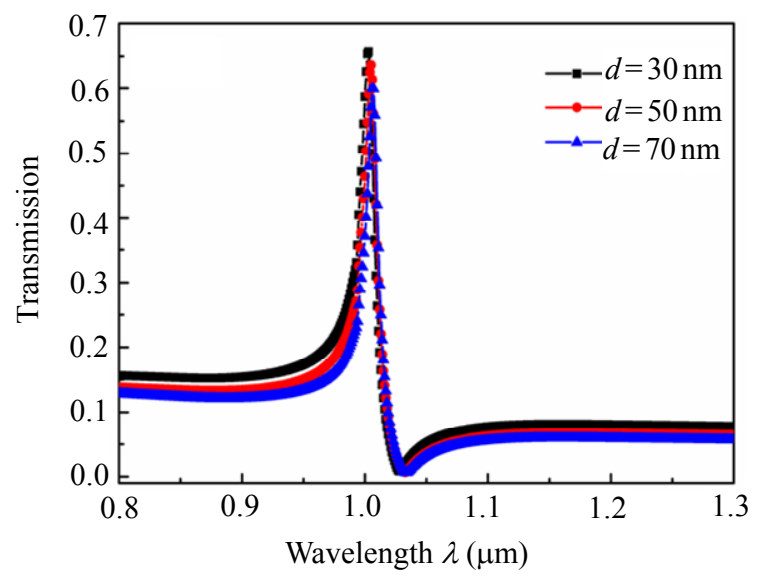

(a)

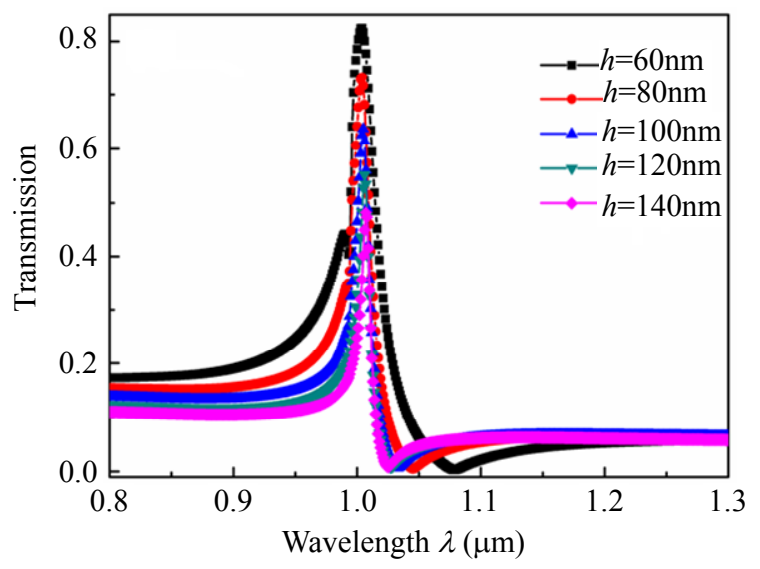

(b)

Fig. 6 Transmission spectra of the MIM waveguide coupled with two rings and rectangular cavities with (a) changing $d(h=$ $100 \mathrm{~nm})$ and (b) changing $h(d=50 \mathrm{~nm})$.

Finally, we will discuss the implications of the findings in context of existing research and potential applications of our structure. Since the wavelengths of SPPs corresponding to the transmission peaks are allowed to transport efficiently, while others are forbidden. Our structure can be used as SPPs band-pass filters, which is very different from the 
band-stop filters in previous researches. The positions of transmission peaks have a linear relationship with the refraction index of the material under sensing. In addition, the device not only has a small size easily to integration, but it also has a high sensing sensitivity, which has a potential application in designing nanoscale devices.

However, the further work should be conducted, such as how to collect the infrared and visible light signals synchronously, how to integrate the infrared and visible lights, how to further improve the sensitivity of the sensor, and how to improve the quality factor of the sensor.

\section{Conclusions}

We have proposed a plasmonic waveguides coupled with rectangular and two shunt-wound rings resonators for the refractive index sensor. The sensing properties of the proposed sensor are numerically analyzed by using FEM. The positions of the Fano resonance transmission peaks have linear relations with the refractive index of the analyte. With an increase in the refractive index and the rings cavity radius, the Fano resonance peak has a red shift. The maximum sensitivity is $1160 \mathrm{~nm} / \mathrm{RIU}$, and its corresponding sensing resolution is $8.62 \times 10^{-5} \mathrm{RIU}$. It has a relatively high quality factor of 73. For its above-mentioned merits, especially the high $S$ and high $F O M$, the plasmonic refractive index sensor with a simple structure could provide a promising approach for gas detection, biosensors, and medical diagnostics.

\section{Acknowledgement}

The authors thank Xiangxian WANG from the School of Science, Lanzhou University of Technology, Lanzhou, China for their discussions to this research. This work is supported by the National Natural Science Foundation of China (Grant Nos. 61367005 and 74011119) and the Natural Science Foundation of Gansu Province (Grant No. 17JR5RA078).
Open Access This article is distributed under the terms of the Creative Commons Attribution 4.0 International License (http://creativecommons.org/licenses/by/4.0/), which permits unrestricted use, distribution, and reproduction in any medium, provided you give appropriate credit to the original author(s) and the source, provide a link to the Creative Commons license, and indicate if changes were made.

\section{References}

[1] V. V. Temnov, G. Armelles, U. Woggon, D. Guzatov, A. Cebollada, A. G. Martin, et al., "Active magneto-plasmonics in hybrid metal-ferromagnet structures," Nature Photonics, 2010, 4(2): 107-111.

[2] S. I. Bozhevolnyi, V. S. Volkov, E. Devaux, J. Y. Laluet, and T. W. Ebbesen, "Channel plasmon subwavelength waveguide components including interferometers and ring resonators," Nature, 2006, 440(7083): 508-511.

[3] H. Lu, X. M. Liu, D. Mao, and G. X. Wang, "Plasmonic nanosensor based on Fano resonance in waveguide-coupled resonators," Optics Letters, 2012, 37(18): 3780-3782.

[4] C. Zhao and Y. Li, "Multiple Fano resonances based on different waveguide modes in a symmetry breaking plasmonic system," IEEE Photonics Journal, 2014, 6(6): 4802208-1-4802208-8.

[5] N. Park, S. Yu, and X. Piao, "Control of Fano asymmetry in plasmon induced transparency and its application to plasmonic waveguide modulator," Optics Express, 2012, 20(17): 18994-18999.

[6] D. M. Becerra, J. B. G. Díaz, V. V. Temnov, A. Cebollada, G. Armelles, T. Thomay, et al., "Enhancement of the magnetic modulation of surface plasmon polaritons in $\mathrm{Au} / \mathrm{Co} / \mathrm{Au}$ films," Applied Physics Letter, 2010, 97(18): 183114-1-183114-3.

[7] K. H. Wen, Y. H. Hu, L. Chen, J. Y. Zhou, L. Lei, and Z. M. Meng, "Single/dual Fano resonance based on plasmonic metal-dielectric-metal waveguide," Plasmonics, 2016, 11(1): 315-321.

[8] H. N. Daghestani and B. W. Day, "Theory and applications of surface plasmon resonance, resonant mirror, resonant waveguide grating, and dual polarization interferometry biosensors," Sensors, 2010, 10(11): 9630-9646.

[9] W. S. Yue, Z. H. Wang, Y. Yang, L. Q. Chen, A. Syed, K. Wang, et al., "Electron-beam lithography of gold nanostructures for surface-enhanced Raman scattering," Journal of Micro-Mechanics \& Microengineering, $\quad 2012, \quad 22(12): \quad 125007-1-$ 125007-9.

[10] B. Ni, X. Y. Chen, D. Y. Xiong, H. Liu, G. H. Hua, J. H. Chang, et al., "Infrared plasmonic refractive index-sensitive nanosensor based on electromagnetically induced transparency of 
waveguide resonator systems," Optical \& Quantum Electronics, 2015, 47(6): 1339-1346.

[11] R. Kirchain and L. Kimerling, "A roadmap for nanophotonics," Nature Photonics, 2007, 1(6): 303-305.

[12] L. J. Liu, S. L. He, and Z. H. Han, "Novel surface plasmon waveguide for high integration," Optics Express, 2005, 13(17): 6645-6650.

[13] Q. Zhang, X. G. Huang, X. S. Lin, J. Tao, and X. P. Jin, "A subwavelength coupler-type MIM optical filter," Optics Express, 2009, 17(9): 7533-7539.

[14] G. X. Wang, H. Lu, and X. M. Liu, "Dispersionless slow light in MIM waveguide based on a plasmonic analogue of electromagnetically induced transparency," Optics Express, 2012, 20(19): 20902-20907.

[15] J. J. Chen, Z. Li, S. Yue, J. H. Xiao, and Q. H. Gong, "Plasmon-induced transparency in asymmetric T-shape single slit," Nano Letters, 2012, 12(5): 2494-2498.

[16] J. Park, H. Kim, and B. Lee, "High order plasmonic Bragg reflection in the metal-insulator-metal waveguide Bragg grating," Optics Express, 2008, 16(1): 413-425.

[17] H. W. Zhao, "Surface plasmon polaritons based optical directional coupler," Science in China, 2008, 40(10): 3025-3029.

[18] P. Fan, Z. Yu, S. Fan, and M. L. Brongersma, "Optical Fano resonance of an individual semiconductor nanostructure," Nature Materials, 2014, 13(5): 471-475.

[19] M. J. Sung, "Approach the angular sensitivity limit in surface plasmon resonance sensors with low index prism and large resonant angle," Optical Engineering, 2010, 49(49): 054403-1-054403-6.

[20] Y. Shen, J. H. Zhou, T. R. Liu, Y. T. Tao, R. B. Jiang, M. X. Liu, et al., "Plasmonic gold mushroom arrays with refractive index sensing figures of merit approaching the theoretical limit," Nature Communications, 2013, 4(4): 3381-1-3381-9.

[21] Y. Tang, Z. D. Zhang, R. B. Wang, Z. Y. Hai, C. Y. Cue, W. D. Zhang, et al., "Refractive index sensor based on Fano resonances in metal-insulator-metal waveguides coupled with resonators," Sensors, 2017, 17(4): 784-1-784-8

[22] W. Wei, X. Zhang, and X. M. Ren, "Plasmonic circular resonators for refractive index sensors and filters," Nanoscale Research Letters, 2015, 10(1): $1-6$.

[23] H. F. Gai, J. Wang, and Q. Tian, "Modified Debye model parameters of metals applicable for broadband calculations," Applied Optics, 2007, 46(12): 2229-2233.

[24] Z. D. Zhang, L. Liang, C. Y. Xue, W. D. Zhang, and S. B. Yan, "Fano resonance based on metal-insulator-metal waveguide-coupled double rectangular cavities for plasmonic nanosensors," Sensors, 2016, 16(5): 642-1-642-10.

[25] R. D. Kekatpure, A. C. Hryciw, E. S. Barnard, and M. L. Brongersma, "Solving dielectric and plasmonic waveguide dispersion relations on a pocket calculator," Optics Express, 2009, 17(26): 24112-24129.

[26] J. H. Zhu, Q. J. Wang, P. Shum, and X. G. Huang, “A simple nanometeric plasmonic narrow-band filter structure based on metal-insulator-metal waveguide," IEEE Transactions on Nanotechnology, 2011, 10(6): 1371-1376.

[27] F. F. Hu, H. X. Yi, and Z. P. Zhou, "Wavelength demultiplexing structure based on arrayed plasmonic slot cavities," Optics Letters, 2011, 36(8): 1500-1502.

[28] Q. Li, T. Wang, Y. K. Su, M. Yan, and M. Qiu, "Coupled mode theory analysis of mode-splitting in coupled cavity system," Optics Express, 2010, 18(8): 8367-8382.

[29] X. J. Piao, S. Yu, S. Koo, K. Lee, and N. Park, "Fano-type spectral asymmetry and its control for plasmonic metal-insulator-metal stub structures," Optics Express, 2011, 19(11): 10907-10912.

[30] R. Ameling, L. Langguth, M. Hentschel, and M. Mesch, "Cavity-enhanced localized plasmon resonance sensing," Applied Physics Letters, 2010, 97(25): 253116-1-253116-3. 